03

\title{
Конкуренция режимов колебаний плохо обтекаемого тела в воздушном потоке
}

\author{
(C) А.Н. Рябинин, Р.В. Шмигирилов \\ Санкт-Петербургский государственный университет, \\ 199034 Санкт-Петербург, Россия \\ e-mail: a.ryabinin@spbu.ru
}

Поступило в Редакцию 4 декабря 2020 г.

В окончательной редакции 4 декабря 2020 г.

Принято к публикации 8 декабря 2020 г.

На основе известных математических моделей, описывающих колебания в потоке газа плохо обтекаемого тела с одной степенью свободы, предложена модель колебаний тела с двумя степенями свободы. Составлены уравнения поперечных поступательных колебаний и вращательных колебаний упруго закрепленного тела вокруг оси, перпендикулярной вектору скорости набегающего потока. Методом Крылова-Боголюбова в первом приближении уравнения сводятся к уравнениям для медленно меняющихся амплитуд и частот колебаний. Оказалось, что дифференциальные уравнения, выписанные для квадратов безразмерных амплитуд поступательных и вращательных колебаний, совпадают с известными уравнениями Лотки-Вольтерры, описывающими конкуренцию между двумя видами животных, питающихся одинаковой пищей. Коэффициенты уравнений зависят от скорости набегающего потока. Модель верифицирована на примере колебаний макета сегмента моста в аэродинамической трубе.

Ключевые слова: поступательные и вращательные колебания, воздушный поток, плохо обтекаемое тело.

DOI: $10.21883 /$ JTF.2021.05.50686.335-20

\section{Введение}

Плохо обтекаемые упругие или упруго закрепленные тела под действием ветра могут совершать поступательные или вращательные колебания. Источником колебаний протяженных тел могут быть периодически сходящие вихри, образующие цепочку Кармана, если частота вихрей близка к собственной частоте колебаний конструкции. В настоящей работе рассматриваются колебания, называемые галопированием [1]. При этом принимается, что частота периодического схода вихрей намного превышает собственную частоту колебаний. Поэтому аэродинамические силы, возникающие вследствие схода вихрей, осредняются и не оказывают влияния на существенно медленный процесс колебания при галопировании. В большинстве работ, посвященных колебаниям мостов, неустойчивости, приводящие к галопированию, рассматривались для определения критической скорости ветра в соответствии с линейной динамической теорией [2]. Предполагалось, что при превышении критической скорости воздушного потока колебания интенсивно нарастают и неизбежно приводят к катастрофе. Однако в закритической области мосты могут совершать колебания с ограниченной амплитудой из-за аэродинамических нелинейностей, и амплитуды посткритических колебаний в определенном диапазоне скоростей ветра могут быть допустимыми [3]. Настоящая работа посвящена построению математической модели поступательных и вращательных колебаний плохо обтекаемого тела и проверке этой модели на примере колебаний макета сегмента моста, закрепленного на упругой подвеске в рабочей части аэродинамической трубы.

Для описания поступательного галопирования плохо обтекаемого тела в работе [4] предложена квазистационарная модель. В основе этой модели лежит предположение, что аэродинамические силы, действующие на тело, зависят только от относительной скорости потока и от углов, описывающих ориентацию тела относительно вектора скорости воздушного потока. Для поперечного обтекания воздушным потоком протяженного тела, совершающего колебания в направлении, перпендикулярном потоку, нормальная аэродинамическая сила, действующая в направлении движения, зависит только от мгновенного угла атаки $\alpha$. Коэффициент нормальной аэродинамической силы $c_{y}$ в зависимости от угла атаки $\alpha$ можно определить в аэродинамической трубе в опытах с неподвижно закрепленным телом. В работе [4] зависимость $c_{y}(\alpha)$ квадратной призмы аппроксимировали полиномом пятого порядка. Позже [5] было установлено, что модель работает лучше, если аппроксимировать зависимость $c_{y}(\alpha)$ полиномом седьмого порядка. Квазистационарная модель широко использовалась в дальнейшем для описания поступательного галопирования прямоугольных цилиндров различных пропорций [6], цилиндров с треугольным поперечным сечением [7], ромбовидным поперечным сечением [8], призм конечного удлинения [9].

Модель колебаний упруго закрепленного тела с двумя степенями свободы разрабатывалась в работах $[10,11]$. Авторами этих работ была сделаны попытки распространить квазистационарную модель галопирования на 
вращательные колебания двух плохо обтекаемых тел: квадратной призмы и углового профиля. Трудность применения этих моделей заключается в том, что разные точки вращающегося тела имеют разную скорость и, следовательно, мгновенный угол атаки этих точек различен. Приходится выбирать характерную точку для определения мгновенного угла атаки, в этом заключается некоторый произвол.

\section{1. Математическая модель поступательных и вращательных колебаний плохо обтекаемого тела}

Пусть тело закреплено на упругой подвеске с одной степенью свободы. В неподвижной системе координат оно может перемещаться вдоль оси $O Y$, перпендикулярной скорости набегающего потока $v$ (рис. 1). Тело наклонено под углом $\theta$ к горизонту.

Скорость тела относительно среды складывается из двух перпендикулярных векторов. Первый вектор направлен вдоль оси $O X$, его абсолютная величина равна $v$. Второй вектор представляет собой скорость движения тела вдоль оси $O Y$. Его величина равна $\dot{y}$, где $y-$ координата центра масс тела, точка над символом здесь и далее обозначает дифференцирование по времени. Угол атаки $\alpha$ (угол между осью $O X$ и осью скоростной системы координат $\left.O X_{a}\right)$ и относительная скорость тела $v_{r}$ будут определяться формулами

$$
\begin{gathered}
\alpha=\theta-\operatorname{arctg}(\dot{y} / v), \\
v_{r}=v\left(1+(\dot{y} / v)^{2}\right)^{0.5} \approx v\left(1+0.5(\dot{y} / v)^{2}\right) .
\end{gathered}
$$

Далее будем считать углы атаки малыми и пользоваться приближенными выражениями: $\alpha=\theta-(\dot{y} / v), v_{r}=v$.

Коэффициент $c_{y}$ силы, действующей вдоль оси $O Y$, аппроксимируют полиномом степени $n$ по углу атаки $\alpha$. Не нарушая общности задачи, примем, что коэффициент $A_{0}$ при $\alpha^{0}$ равен нулю, поскольку от величины этого

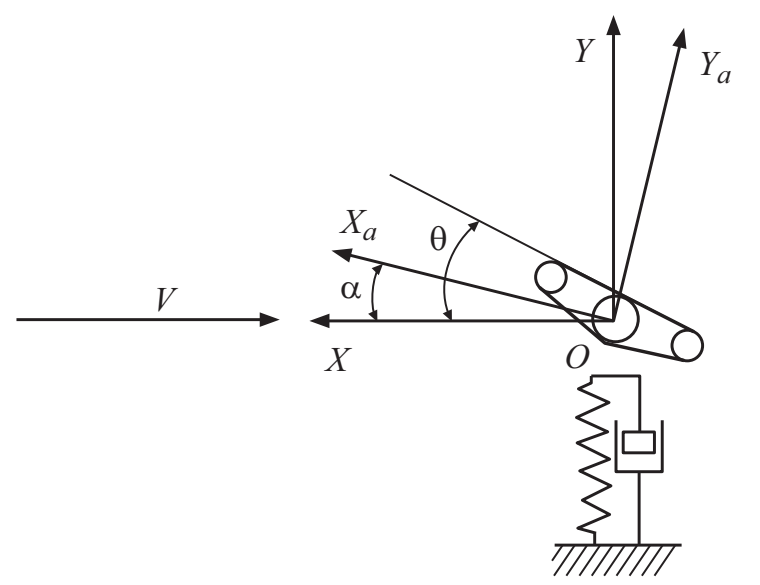

Рис. 1. Моделирование поступательного галопирования. члена зависит только среднее значение координаты $y=y_{0}$. Уравнение движения запишется в виде

$$
m \ddot{y}+r_{1} \dot{y}+k_{1} y=(1 / 2) s \rho_{0} v^{2} \sum_{i=1}^{n} A_{i} \alpha^{i},
$$

где $m-$ масса тела, $r_{1}-$ коэфффициент трения в упругой подвеске, $k_{1}$ - приведенная жесткость подвески, $s-$ характерная площадь тела, $\rho_{0}-$ плотность воздуха.

Введем обозначения:

$$
\begin{gathered}
\mu_{1}=-s \rho_{0} w A_{1} /(2 m), \quad \eta_{1}=r_{1} / m, \\
\omega_{1}^{2}=k_{1} / m, \quad v_{1}=\eta_{1} w / \mu_{1},
\end{gathered}
$$

где $w$ - характерный размер тела. Поступательное галопирование может происходить только при условии $A_{1}<0$ [2], поэтому $\mu_{1}>0$. Пусть $n=3$, тогда

$$
\begin{aligned}
& \ddot{y}+\omega_{1}^{2} y=\mu_{1} \frac{v}{w} \dot{y}\left[1-\frac{v_{1}}{v}+\frac{A_{2}}{A_{1}} \frac{\dot{y}}{v}-2 \frac{A_{2}}{A_{1}} \theta+\frac{A_{3}}{A_{1}}\left(\frac{\dot{y}}{v}\right)^{2}\right. \\
& \left.-3 \frac{A_{3}}{A_{1}}\left(\frac{\dot{y}}{v}\right) \theta+3 \frac{A_{3}}{A_{1}} \theta^{2}\right]-\mu_{1} \frac{v^{2}}{w}\left(\theta-\frac{A_{2}}{A_{1}} \theta^{2}+\frac{A_{3}}{A_{1}} \theta^{3}\right) .
\end{aligned}
$$

Эксперименты показывают, что колебания при галопировании близки к гармоническим и происходят с частотой, близкой к собственной частоте конструкции. Параметр $\mu_{1}$ в правой части уравнения мал.

Для моделирования вращательных колебаний за основу была взята модель колебаний цилиндра малого удлинения, предложенная в работе [12]. Уравнение вращательных колебаний тела с моментом инерции $I_{z}$ вокруг оси $O Z$, проходящей через центр масс, имеет вид

$$
I_{z} \ddot{\theta}+r_{2} \dot{\theta}+k_{2} \theta=s w^{2} \frac{\rho_{0} v}{2} m_{\dot{\alpha}}\left(1-\delta \alpha^{2}\right) \dot{\alpha},
$$

где $r_{2}-$ коэффициент трения в подвеске, $k_{2}-$ жесткость упругой подвески, $m_{\dot{\alpha}}-$ вращательная производная момента тангажа, $\delta$ - параметр, который определяется в эксперименте с вращательными колебаниями по амплитуде вращательных колебаний, к которой асимптотически приближается колебательная система при возрастании скорости потока. При преобразовании уравнения вращательного колебательного движения нужно продифференцировать выражение для угла атаки $\alpha=\theta-(\dot{y} / v)$. Из уравнения (1) следует, что $\ddot{y} \approx-\omega_{1}^{2} y$. С использованием обозначений

$$
\begin{gathered}
\mu_{2}=s \rho_{0} w^{3} m_{\dot{\alpha}} /\left(2 I_{z}\right), \quad \eta_{2}=r_{2} / I_{z}, \\
\omega_{2}^{2}=k_{2} / I_{z}, \quad v_{2}=\eta_{2} w / \mu_{2}
\end{gathered}
$$

уравнение вращательных колебаний примет вид

$$
\begin{aligned}
\ddot{\theta}+\omega_{2}^{2} \theta= & \mu_{2} \frac{v}{w}\left[1-\delta \theta^{2}-\delta\left(\frac{\dot{y}}{v}\right)^{2}+2 \delta \theta \frac{\dot{y}}{v}\right] \\
& \times\left(\dot{\theta}+\frac{\omega_{1}^{2} y}{v}\right)-\mu_{2} v_{2} \dot{\theta} / w .
\end{aligned}
$$


Система уравнений (1) и (2) описывает два связанных осциллятора. Нелинейные члены, находящиеся в правых частях уравнений, умножены на малые параметры $\mu_{1}$ и $\mu_{2}$. Поэтому для решения системы можно применять метод Крылова-Боголюбова [13]. Предположим, что отношение частот $\omega_{1} / \omega_{2}$ не равно единице и не отличается от единицы на малую величину.

Введем новые переменные амплитуды $\rho_{y}, \rho_{\theta}$ и фазы $\psi_{1}, \psi_{2}$ колебаний

$$
\begin{array}{ll}
y=\rho_{y} \cos \psi_{1}, & \psi_{1}=\omega_{1} t+\varphi_{1}, \\
\theta=\rho_{\theta} \cos \psi_{2}, & \psi_{2}=\omega_{2} t+\varphi_{2} .
\end{array}
$$

Амплитуды $\rho_{y}, \rho_{\theta}$ и сдвиги фаз $\varphi_{1}, \varphi_{2}$ являются медленными функциями времени. Обозначим правые части уравнений (1) и (2) $\mu_{1} f_{1}$ и $\mu_{2} f_{2}$ соответственно. В первом приближении метода Крылова-Боголюбова для получения выражений для производных амплитуды и фазы выполняется осреднение по времени с использованием формул:

$$
\dot{\rho}_{y}=-\frac{\mu_{1}}{2 \pi m \omega_{1}} \int_{0}^{2 \pi m} f_{1}\left(\rho_{y} \cos \psi_{1},-\rho_{y} \omega_{1} \sin \psi_{1}, \rho_{\theta} \cos \psi_{2},\right.
$$$$
\left.-\rho_{\theta} \omega_{2} \sin \psi_{2}\right) \sin \psi_{1} d \psi_{1},
$$

$\dot{\rho}_{\theta}=-\frac{\mu_{2}}{2 \pi m \omega_{2}} \int_{0}^{2 \pi m} f_{2}\left(\rho_{y} \cos \psi_{1},-\rho_{y} \omega_{1} \sin \psi_{1}, \rho_{\theta} \cos \psi_{2}\right.$,

$$
\left.-\rho_{\theta} \omega_{2} \sin \psi_{2}\right) \sin \psi_{2} d \psi_{2}
$$

$$
\rho_{y} \dot{\psi}_{1}=\rho_{y} \omega_{1}-\frac{\mu_{1}}{2 \pi m \omega_{1}} \int_{0}^{2 \pi m} f_{1}\left(\rho_{y} \cos \psi_{1},-\rho_{y} \omega_{1} \sin \psi_{1}\right. \text {, }
$$

$\left.\rho_{\theta} \cos \psi_{2},-\rho_{\theta} \omega_{2} \sin \psi_{2}\right) \cos \psi_{1} d \psi_{1}$.

$$
\rho_{\theta} \dot{\psi}_{2}=\rho_{\theta} \omega_{2}-\frac{\mu_{1}}{2 \pi m \omega_{2}} \int_{0}^{2 \pi m} f_{2}\left(\rho_{y} \cos \psi_{1},-\rho_{y} \omega_{1} \sin \psi_{1},\right.
$$

$\left.\rho_{\theta} \cos \psi_{2},-\rho_{\theta} \omega_{2} \sin \psi_{2}\right) \cos \psi_{2} d \psi_{2}$.

Каждая из функции $f_{1}$ и $f_{2}$ представляет собой сумму слагаемых, которые состоят из произведений синусов и косинусов $\psi_{1}$ и $\psi_{2}$ в некоторой степени.

После вычисления интегралов в выражениях (3) и (4) получаем приближенные уравнения для амплитуд поступательных и вращательных колебаний:

$$
\begin{gathered}
\dot{\rho}_{y}=\frac{\mu_{1}}{2} \frac{v}{w} \rho_{y}\left\{1-\frac{v_{1}}{v}+\frac{3 A_{3}}{4 A_{1}}\left[\left(\frac{\rho_{y} \omega_{1}}{v}\right)^{2}+2 \rho_{\theta}^{2}\right]\right\}, \\
\dot{\rho}_{\theta}=\frac{\mu_{2}}{2} \frac{v}{w} \rho_{\theta}\left\{1-\frac{v_{2}}{v}-\frac{\delta}{4}\left[\rho_{\theta}^{2}+2\left(\frac{\rho_{y} \omega_{1}}{v}\right)^{2}\right]\right\} .
\end{gathered}
$$

Таким же образом вычислив интегралы в формулах (5) и (6), можно получить уравнения для фаз колебаний. Однако в дальнейшем уравнения для фаз не понадобятся, поэтому они не приводятся. Приведя к безразмерному виду амплитуды поступательных колебаний $\rho_{Y}=\rho_{y} \omega_{1} / v$ и используя обозначения $b_{1}=-3 A_{3} /\left(4 A_{1}\right)$ и $b_{2}=\delta / 4$, получим

$$
\begin{aligned}
& \dot{\rho}_{Y}=\frac{\mu_{1}}{2} \frac{v}{w} \rho_{Y}\left[1-\frac{v_{1}}{v}-b_{1}\left(\rho_{Y}^{2}+2 \rho_{\theta}^{2}\right)\right], \\
& \dot{\rho}_{\theta}=\frac{\mu_{2}}{2} \frac{v}{w} \rho_{\theta}\left[1-\frac{v_{2}}{v}-b_{2}\left(\rho_{\theta}^{2}+2 \rho_{Y}^{2}\right)\right] .
\end{aligned}
$$

Удобно перейти от уравнений для амплитуд к уравнениям для квадратов амплитуд

$$
\Upsilon=\rho_{Y}^{2}, \quad \Theta=\rho_{\theta}^{2} .
$$

Полученные уравнения совпадают с известными уравнениями Лотки-Вольтерры [14], полученными для описания конкуренции между двумя видами животных, питающихся одинаковой пищей

$$
\begin{aligned}
& \dot{\Upsilon}=\mu_{1} \frac{v}{w} \Upsilon\left[1-\frac{v_{1}}{v}-b_{1}(\Upsilon+2 \Theta)\right], \\
& \dot{\Theta}=\mu_{2} \frac{v}{w} \Theta\left[1-\frac{v_{2}}{v}-b_{2}(\Theta+2 \Upsilon)\right] .
\end{aligned}
$$

В отличие от оригинальных уравнений Лотки-Вольтерры коэффициенты в полученных уравнениях (7) и (8) зависят от скорости набегающего потока. Квадрат амплитуды колебаний соответствуют численности животных.

Система уравнений (7) и (8) симметрична относительно входящих в систему переменных. Положим для определенности, что $v_{1}<v_{2}$. Для нахождения решений уравнений, описывающих колебания с установившейся постоянной амплитудой, нужно приравнять производные по времени в левой части уравнений нулю. Система превратится в алгебраическую. Существует 4 стационарных решения уравнений

1. $\Upsilon=0, \Theta=0$.

2. $\Upsilon=\left(v-v_{1}\right) /\left(b_{1} v\right), \Theta=0$.

3. $\Upsilon=0, \Theta=\left(v-v_{2}\right) /\left(b_{2} v\right)$.

4. $\Upsilon=\left[2 b_{1}\left(v-v_{2}\right)-b_{2}\left(v-v_{1}\right)\right] /\left(3 b_{1} b_{2} v\right)$, $\Theta=\left[2 b_{2}\left(v-v_{1}\right)-b_{1}\left(v-v_{2}\right)\right] /\left(3 b_{1} b_{2} v\right)$.

Для определения устойчивости решений используем линеаризованные уравнения в окрестности решений:

$$
\begin{aligned}
& \left(\begin{array}{l}
\dot{\Upsilon} \\
\dot{\Theta}
\end{array}\right)=\frac{v}{w} \times \\
& \left(\begin{array}{cc}
\mu_{1}\left(1-v_{1} / v-2 b_{1} \Upsilon-2 b_{1} \Theta\right) & -\mu_{1} 2 b_{1} \Upsilon \\
-\mu_{2} 2 b_{2} \Theta & \mu_{2}\left(1-v_{2} / v-2 b_{2} \Upsilon-2 b_{2} \Theta\right)
\end{array}\right) \\
& \times\left(\begin{array}{c}
\Delta \Upsilon \\
\Delta \Theta
\end{array}\right) .
\end{aligned}
$$

Составим характеристическое уравнение для определения собственных чисел матрицы Якоби:

$$
\begin{gathered}
\left|\begin{array}{cc}
\mu_{1}\left(1-v_{1} / v-2 b_{1} \Upsilon-2 b_{1} \Theta\right)-\lambda & -\mu_{1} 2 b_{1} \Upsilon \\
-\mu_{2} 2 b_{2} \Theta & \mu_{2}\left(1-v_{2} / v-2 b_{2} \Upsilon-2 b_{2} \Theta\right)-\lambda
\end{array}\right| \\
=0 .
\end{gathered}
$$


Отрицательные значения собственных чисел указывают на устойчивость решения. Решение неустойчиво, если хотя бы одно собственное число положительно.

Собственные числа матрицы для решения 1 :

$$
\lambda_{1}=\mu_{1}\left(v-v_{1}\right) / v, \quad \lambda_{2}=\mu_{2}\left(v-v_{2}\right) / v .
$$

Решение устойчиво в диапазоне $v<v_{1}$.

Собственные числа матрицы для решения 2:

$$
\begin{gathered}
\lambda_{1}=-\mu_{1}\left(v-v_{1}\right) / v \\
\lambda_{2}=\mu_{2} / v\left[v-v_{2}-2\left(b_{2} / b_{1}\right)\left(v-v_{1}\right)\right] .
\end{gathered}
$$

Если $b_{2} / b_{1}>1 / 2$, решение устойчиво в диапазоне $v>v_{1}$. Если $b_{2} / b_{1}<1 / 2$, решение устойчиво в диапазоне $v_{1}<v<v_{3}$, где $v_{3}=v_{2}+2 b_{2}\left(v_{2}-v_{1}\right) /\left(b_{1}-2 b_{2}\right)$.

Собственные числа матрицы для решения 3 :

$$
\begin{gathered}
\lambda_{1}=\left(\mu_{1} / v\right)\left[v-v_{1}-2\left(b_{1} / b_{2}\right)\left(v-v_{2}\right)\right], \\
\lambda_{2}=-\mu_{2}\left(v-v_{2}\right) / v .
\end{gathered}
$$

Если $b_{2} / b_{1}<2$, решение устойчиво в диапазоне $v>v_{4}$, где $\quad v_{4}=\left(v_{1} b_{2}-2 v_{2} b_{1}\right) /\left(b_{2}-2 b_{1}\right)=v_{2}+b_{2}\left(v_{2}-v_{1}\right)$ $/\left(2 b_{1}-b_{2}\right)$, иначе решение неустойчиво.

Решение 4 существует в диапазоне $v_{4}<v<v_{3}$. Применение критерия Рауса-Гурвица позволяет установить, что оно неустойчиво.

Рассмотрим последовательность смены режимов с постоянной амплитудой колебаний, которую дает построенная модель, при изменении скорости воздушного потока, если коэффициенты удовлетворяют условию $b_{2} / b_{1}<1 / 2$. Пока скорость набегающего потока не превышает критической скорости возникновения поступательных колебаний $v<v_{1}$, колебания отсутствуют. При увеличении скорости потока от $v_{1}$ до $v_{3}$ существуют поступательные колебания, которые при превышении верхней границы $v_{3}$ сменяются вращательными. При последующем уменьшении скорости обратный переход к поступательным колебаниям осуществляется при скорости потока $v_{4}<v_{3}$. При дальнейшем уменьшении скорости колебания прекращаются при скорости, которая равна скорости возникновения колебаний $v_{1}$. Таким образом, модель предсказывает гистерезис при увеличении и уменьшении скорости воздушного потока.

Для некоторых тел лучшие предсказания для амплитуд галопирования дает аппроксимация коэффициента нормальной силы $c_{y}$ полиномом более высокого порядка. Например, для призмы с квадратным поперечным сечением установлено, что лучшие результаты дает аппроксимация $c_{y}$ полиномом седьмого порядка [5] по степеням угла атаки. При этом установлено, что может существовать еще один гистерезис в области критической скорости возникновения поступательных колебаний.

Пусть $n=7$. Тогда метод Крылова-Боголюбова в первом приближении дает вместо системы уравнений (7) и (8) следующую систему

$$
\begin{aligned}
\dot{\Upsilon}= & \mu_{1} \frac{v}{w} \Upsilon\left[1-\frac{v_{1}}{v}+\frac{3 A_{3}}{4 A_{1}}(\Upsilon+2 \Theta)\right. \\
& +\frac{5 A_{5}}{8 A_{1}}\left(\Upsilon^{2}+6 \Upsilon \Theta+3 \Theta^{2}\right) \\
& \left.+\frac{35 A_{7}}{64 A_{1}}\left(\Upsilon^{3}+12 \Upsilon^{2} \Theta+18 \Upsilon \Theta^{2}+4 \Theta^{3}\right)\right], \\
\dot{\Theta} & =\mu_{2} \frac{v}{w} \Theta\left[1-\frac{v_{2}}{v}-b_{2}(\Theta+2 \Upsilon)\right] .
\end{aligned}
$$

Удобно отыскивать установившиеся колебательные режимы тела на упругой подвеске, решая систему обыкновенных дифференциальных уравнений (9) и (10) численно. Мы использовали для решения системы метод Рунге-Кутты четвертого порядка.

\section{2. Экспериментальная проверка математической модели}

Изложенная выше математическая модель проверялась в аэродинамической трубе АТ-12 Санкт-Петербургского университета на примере испытаний модели сегмента моста. Модель моста имела ширину $w=110 \mathrm{~mm}$ и длину $L=780 \mathrm{~mm}$. Мост представляет собой три балки круглого сечения, соединенные перемычками и покрытые настилом. Схема конструкции моста представлена на рис. 2.

Все эксперименты проводились в присутствии концевых шайб круглой формы, имеющих диаметр $200 \mathrm{~mm}$. Назначение концевых шайб - исключение перетекания воздушного потока через торцы модели. Было показано, что в случае моделирования вращательных колебаний толстой пластины соотношение сторон пластины $L / w$ должно быть больше пяти [15]. Пропорции нашей модели удовлетворяют этому требованию.

Коэффициенты $A_{i}(i=0,1, \ldots, 7)$ в разложении $c_{y}$ по степеням угла атаки $\alpha$ определялись по результатам измерения лобового сопротивления и подъемной силы, действующих на модель, неподвижно закрепленную в рабочей части аэродинамической трубы. График зависимости $c_{y}(\alpha)$ приведен на рис. 3. По оси абсцисс отложено отклонение от угла атаки $\alpha_{0}=0.1 \mathrm{rad}$. Ось $O Y$

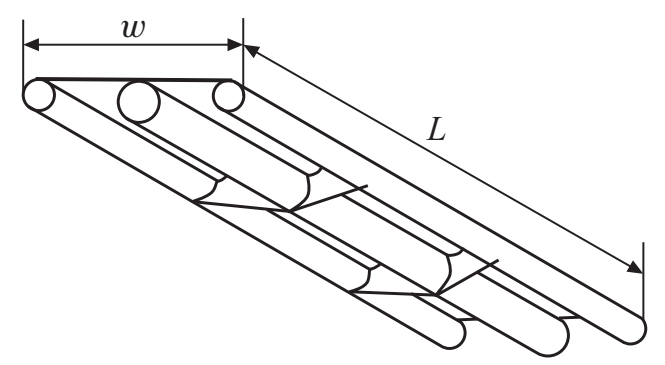

Рис. 2. Схема испытываемого сегмента моста. 


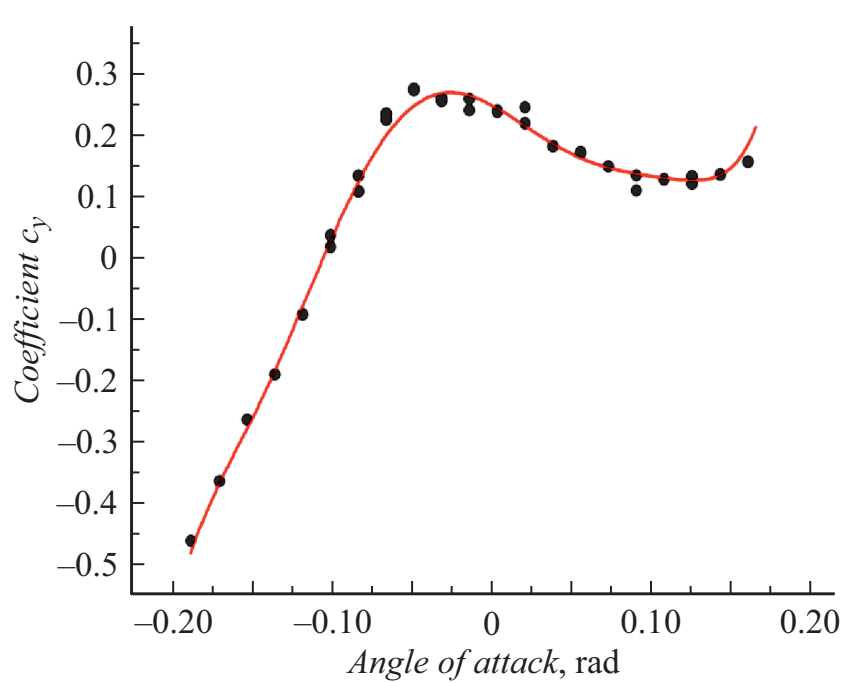

Рис. 3. Экспериментальная зависимость $c_{y}$ от угла атаки $\alpha$ и ее аппроксимация полиномом 7-го порядка.

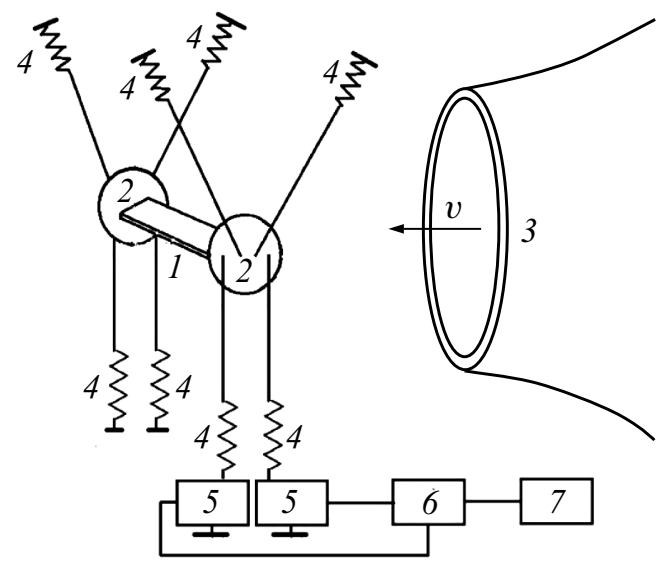

Рис. 4. Схема эксперимента. 1 - модель, $2-$ концевые шайбы, 3 - сопло аэродинамической трубы, 4 - пружины, 5 - полупроводниковые тензопреобразователи, 6 - PC-осциллограф, 7 - компьютер.

перпендикулярна вектору скорости набегающего потока, если угол атаки $\alpha=\alpha_{0}$.

Коэффициенты разложения приводятся ниже:

$$
\begin{aligned}
\left(A_{0}, A_{1}, \ldots, A_{7}\right)= & (0.253,-1.501,-16.96,303,-120 \\
& -12066,10726,185574)
\end{aligned}
$$

Схема эксперимента по колебанию сегмента моста приведена на рис. 4. Модель закреплена в рабочей части аэродинамической трубы с помощью восьми стальных пружин и проволочных тяг, соединяющих пружины с моделью. Установленная таким образом модель могла перемещаться с шестью степенями свободы, однако в эксперименте наблюдались только поступательные колебания вдоль вертикальной оси $O Y$ и вращательные колебания вокруг оси $O Z$.
Два полупроводниковых тензопреобразователя C-50 регистрируют натяжение двух нижних пружин. PCосциллограф Velleman-PCS500 преобразует аналоговые выходные сигналы тензопреобразователей в цифровые и передает их на управляющий компьютер. Частота считывания показаний составляет $1250 \mathrm{~Hz}$. Длительность записи показаний равна $3.3 \mathrm{~s}$.

Процедура калибровки и последующая обработка результатов позволяют связать амплитуды колебаний выходного напряжения тензопреобразователей с амплитудами вращательных и поступательных колебаний сегмента моста.

Установившиеся колебания сегмента моста имели место при небольших положительных значениях угла атаки $\alpha$. Далее мы приводим результаты по измерению амплитуд колебаний при угле атаки, который в отсутствие колебаний был равен $\alpha_{0}=0.1 \mathrm{rad}$. По мере увеличения скорости потока модель проходит через два различных режима колебаний. При малых скоростях возникают поступательные колебания с частотой $2 \mathrm{~Hz}$ в вертикальном направлении. Затем эти колебания сменяются вращательными колебаниями вокруг оси, проходящей через плоскость симметрии модели. Частота поступательных колебаний намного меньше частоты вращательных колебаний $7.5 \mathrm{~Hz}$. Диапазоны существования двух режимов колебаний перекрываются.

На рис. 5 приведены зависимости безразмерных амплитуд колебаний, полученные в эксперименте и в результате численного расчета, проведенного методом Рунге-Кутты. В качестве исходных данных в расчете задавались следующие величины

$$
v_{1}=4.2 \mathrm{~m} / \mathrm{s}, \quad v_{2}=13.5 \mathrm{~m} / \mathrm{s}, \quad b_{2}=3.5 .
$$

Коэффициенты разложения $c_{y}$ по степеням угла атаки $\alpha$, используемые в расчете, приведены выше.

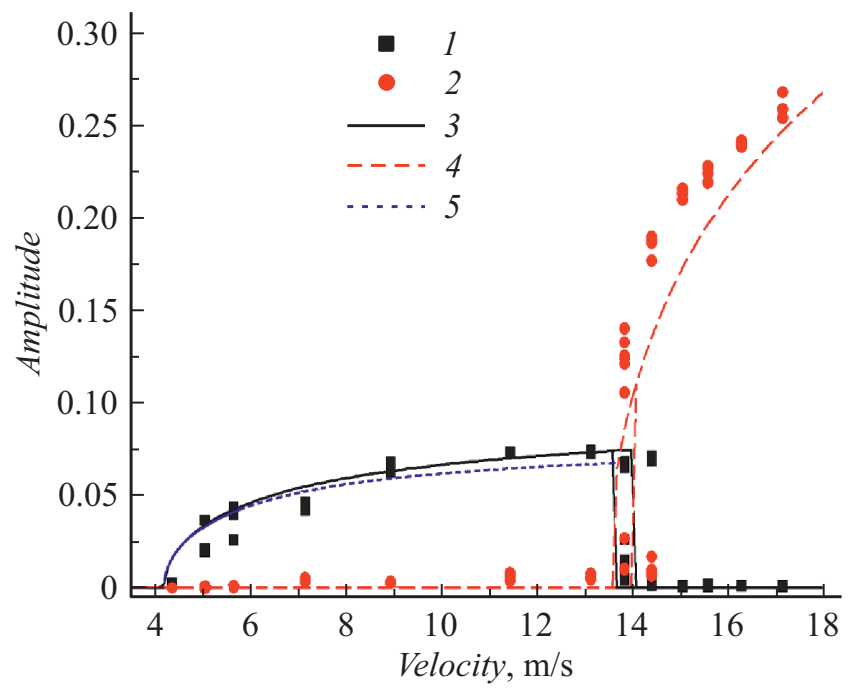

Рис. 5. Зависимости безразмерных амплитуд поступательных $\rho_{Y}$ и вращательных $\rho_{\theta}$ колебаний от скорости набегающего потока: $1-\rho_{Y}$, эксперимент; $2-\rho_{\theta}$, эксперимен; $3-\rho_{Y}$, расчет; $4-\rho_{\theta}$, расчет; $5-\rho_{Y}$, расчет по простой модели. 
Параметр $b_{1}$ простой модели, учитывающей в разложении $c_{y}$ по степеням угла атаки $\alpha$ только члены не выше третьего порядка малости, составил 110.5 . Правая граница области гистерезиса $v_{3}$, рассчитанная по простой модели, равна $14.13 \mathrm{~m} / \mathrm{s}$. Левая граница $v_{4}=13.65 \mathrm{~m} / \mathrm{s}$. В более сложной модели значения границ области гистерезиса отличаются от границ простой модели не более, чем на $0.1 \mathrm{~m} / \mathrm{s}$.

Таким образом, картина смены режимов колебаний качественно описывается предложенной математической моделью. Некоторое количественное расхождение результатов, предсказываемых моделью, и экспериментальных данных можно объяснить погрешностями эксперимента и несовершенством модели, описывающей вращательные колебания.

\section{Заключение}

Предложена математическая модель, описывающая поступательные и вращательные колебания плохо обтекаемого тела в потоке газа. За основу модели поступательного галопирования была взята известная квазистационарная модель, за основу моделирования вращательных колебаний принята модель колебаний цилиндра малого удлинения. При моделировании учитывается, что мгновенный угол атаки складывается из наклона тела и угла, тангенс которого равен отношению вертикальной скорости тела к горизонтальной скорости тела относительно среды. При аппроксимации зависимости коэффициента нормальной силы полиномом третьего порядка уравнения движения тела сводятся к дифференциальным уравнениям, совпадающим с уравнениями Лотки-Вольтерры, первоначально полученными для описания конкуренции двух видов животных, питающихся одинаковой пищей. Предсказания модели подтверждены в эксперименте в аэродинамической трубе. Модель правильно описывает смену режимов колебаний, включая гистерезис, связанный с увеличением и последующим уменьшением скорости воздушного потока.

\section{Финансирование работы}

Финансовая поддержка настоящего проекта обеспечена Российским фондом фундаментальных исследований, грант 19-38-90045.

\section{Конфликт интересов}

Авторы заявляют, что у них нет конфликта интересов.

\section{Список литературы}

[1] Э. Симиу, Р. Сканлан. Воздействие ветра на здания и сооружения (Стройиздат, М., 1984) [Пер. с англ.: Е. Simiu, R. Scanlan. Wind effect on structures: An introduction to wind engineering (John Wiley \& Sons, NY. 1978)]

[2] J.P. Den Hartog. Trans. Am. Inst. Electr. Eng. 51, 1074 (1932).
[3] M. Zhang, F. Xu, Y. Han. J. Wind Eng. Ind. Aerod. 203, 104251 (2020). DOI: 10.1016/j.jweia.2020.104251

[4] G.V. Parkinson, N.P.H. Brooks. J. Appl. Mech., 28, 252 (1961).

[5] G.V. Parkinson, J.D. Smith. Quart. J. Mech Appl. Math., 17, 225 (1964).

[6] M. Novak. ASCE J. Engin. Mech. Div., 95, 115 (1969).

[7] G. Alonso, A. Sanz-Lobera, J. Meseguer. J. Fluids Struct., 33, 243 (2012). DOI: 10.1016/j.jfluidstructs.2012.04.008

[8] G. Alonso, E. Valero, J. Meseguer, European J. Mech. B Fluids., 28, 328 (2009). DOI: 10.1016/j.euromechflu.2008.09.004

[9] В.Д. Люсин, А.Н. Рябинин. Вестн. СПб. ун-та. Сер. 1, 2, 139 (2011).

[10] R.D. Blevins, W.D. Iwan. J. Appl. Mech. 41, 1113 (1974).

[11] Y.M. Desai, A.H. Shah, N. Popplewell. J. Eng. Mech., 116 (12), 2583 (1990).

[12] А.Н. Рябинин, Н.А. Киселев. Вестн. С.-П. ун-та. Сер. 1, 3 (2), 315 (2016). DOI: 10.21638/11701/spbu01.2016.216

[13] Н.Н. Боголюбов, Ю.А. Митропольский. Асимптотические методы в теории нелинейных колебаний (4-е изд. Наука, М., 1974).

[14] J.D. Murray. Mathematical Biology I. An Introduction (Springer-Verlag, New York-Berlin-Heidelberg, 2002).

[15] R. Shmigirilov, A. Ryabinin. In Conference Topical Problems of Fluid Mechanics. Proceedings, ed. by D. Šimurda and T. Bodnár (Institute of Thermomechanics, Prague, 2020). P. 200. DOI: 10.14311/TPFM.2020.026 